\title{
Influence of Menopausal Status on Lipids and Lipoproteins and Fat Mass Distribution: The Pioneer Project
}

Melinda Villarreal, Ronique Pleasant, Nancy M. DiMarco, Barney C. Sanborn, Joshua S. Wooten

Texas Woman's University

Int J Exerc Sci 2(1): S3, 2009. Following menopause, fat redistribution and increased risk for dyslipidemia are common. However, the influence of menopause on the associations between total and regional fat mass with lipids and lipoproteins remains unclear. PURPOSE: The purpose of this investigation was to determine the influence of menopausal status on associations between total and regional fat mass and concentrations of lipids and lipoprotein. METHODS: Healthy non-smoking women $(n=209)$ were grouped based on current menstrual status: premenopausal $(n=143$, mean $\pm S D$; age $=42.7 \pm 7.7 \mathrm{yr}, \mathrm{BMI}=24.5 \pm 4.0 \mathrm{~kg} / \mathrm{m}^{-2}, \mathrm{WC}=77.4 \pm 9.9 \mathrm{~cm}$ ) or postmenopausal $\left(\mathrm{n}=66\right.$, mean $\pm \mathrm{SD}$; age $\left.=52.9 \pm 5.3 \mathrm{yr}, \mathrm{BMI}=24.9 \pm 4.2 \mathrm{~kg} / \mathrm{m}^{-2}, \mathrm{WC}=78.8 \pm 9.9 \mathrm{~cm}\right)$. Fasting $(12$ hr) serum samples were analyzed for concentrations of total cholesterol (TC), triglyceride (TG), LDL-C, HDL-C, HDL2-C, and HDL3-C. Total (TF), abdominal (AF), hip (HF) and mid-thigh (MTF) fat mass were quantified by DXA. A MANCOVA was used to determine differences between groups for total and regional fat mass and concentrations of lipids and lipoproteins controlling for hormone replacement therapy (HRT) status. Stepwise multiple regression analysis determined if menopausal status influenced the association of total and regional fat mass with lipids and lipoproteins. The criterion reference for statistical significance was set at a $P<0.05$. RESULTS: Postmenopausal women had significantly greater concentrations of TC, HDL-C, LDL-C and $\mathrm{HDL}_{3}-\mathrm{C}$ than premenopausal women. No significant differences were observed between groups for total and regional fat mass. In premenopausal women, $\mathrm{AF}$ predicted TC, but no associations were observed in postmenopausal women. In premenopausal women, $\mathrm{AF}+\mathrm{HF}$ and $\mathrm{AF}+\mathrm{TF}$ were significant predictors of TG and LDL$\mathrm{C}$, respectively. In contrast, only AF predicted TG and LDL-C in postmenopausal women. AF+MTF best predicted HDL-C in premenopausal women; however, TF+MTF best predicted HDL-C in postmenopausal women. In premenopausal women, no associations were observed with $\mathrm{HDL}_{2}-\mathrm{C}$ or $\mathrm{HDL}_{3}-\mathrm{C}$. TF and $\mathrm{TF}+\mathrm{MTF}$ were best predictors of $\mathrm{HDL}_{2}-\mathrm{C}$ and $\mathrm{HDL}_{3}-\mathrm{C}$, respectively in postmenopausal women. CONCLUSION: Menopausal status has an effect on concentrations of lipid and lipoprotein-cholesterol, but not on total and regional fat mass. In addition, menopausal status had an influence on the associations of total and regional fat mass with lipids and lipoproteins.

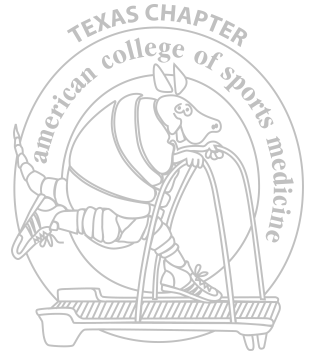

S3 